\title{
A risk model to predict severe postpartum hemorrhage in patients with placenta previa: a single-center retrospective study
}

\author{
Cheng Chen, Xiaoyan Liu, Dan Chen, Song Huang, Xiaoli Yan, Heying Liu, Qing Chang, \\ Zhiqing Liang
}

Department of Gynecology and Obstetrics, the First Affiliated Hospital, Army, Military Medical University, Chongqing 400038, China

Contributions: (I) Conception and design: C Chen, Q Chang, Z Liang; (II) Administrative support: Q Chang; (III) Provision of study materials: C Chen, X Liu, D Chen; (IV) Collection and assembly of data: C Chen, S Huang, X Yan, H Liu; (V) Data analysis and interpretation: C Chen; (VI) Manuscript writing: All authors; (VII) Final approval of manuscript: All authors.

Correspondence to: Qing Chang; Zhiqing Liang. Department of Gynecology and Obstetrics, the First Affiliated Hospital, Army, Military Medical University, Chongqing 400038, China. Email: cqli99@126.com; zhi_lzliang@163.com.

\begin{abstract}
Background: The study aimed to establish a predictive risk model for severe postpartum hemorrhage in placenta previa using clinical and placental ultrasound imaging performed prior to delivery.

Methods: Postpartum hemorrhage patients were retrospectively enrolled. Severe postpartum hemorrhage was defined as exceeding 1,500 mL. Data collected included clinical and placental ultrasound images.

Results: Age of pregnancy, time of delivery, time of miscarriage, history of vaginal delivery, gestational weeks at pregnancy termination, depth of placenta invading the uterine muscle wall were independent risk factors for severe postpartum hemorrhage in placenta previa. A model to predict severe postpartum hemorrhage in placenta previa was established: $\mathrm{P}=\log (\mathrm{Y} / 1-\mathrm{Y})$, where $\mathrm{Y}=-6.942+0.075 X_{1}$ (age) $+1.531 X_{2}$ (times of delivery) $+0.223 X_{3}$ (time of miscarriage) $-3.557 X_{4}$ (vaginal delivery: 1 for yes, 0 for no) $+1.753 X_{5}$ ( 0 for $<37$ weeks, 1 for $\geq 37$ weeks) $+1.574 X_{6}$ (Depth of placenta invading uterine muscle wall: 0 for normal, 1 for placenta adhesion, 2 for placenta implantation, 3 for placenta penetration); discriminant boundary value of the prediction model (probability: P) was 0.268 . Predicting sensitivity $\left(\widehat{S}_{e}\right)=0.765$ (negative predicting accuracy rate), specificity $\left(\widehat{S}_{p}\right)=0.900$ (positive predicting accuracy rate), total accuracy rate $=0.795$, and AUC of ROC curve $=0.8419$.

Conclusions: The risk prediction model which had clinical and ultrasound imaging information prior to delivery had a high decision accuracy. However, before it can be used in the clinic, multicenter large-sample clinical studies should be performed to verify its accuracy and reliability.
\end{abstract}

Keywords: placenta previa; severe postpartum hemorrhage; prediction model; clinical information; placental ultrasound image characteristics

Submitted May 08, 2019. Accepted for publication Aug 12, 2019.

doi: 10.21037/apm.2019.09.04

View this article at: http://dx.doi.org/10.21037/apm.2019.09.04

\section{Introduction}

Postpartum hemorrhage is the primary reason for death in pregnant women. The Society of Obstetrics and Gynecology published the Guideline for Postpartum Hemorrbage to standardize diagnosis and treatment (1-3). Compared to normal postpartum hemorrhage (greater than $500 \mathrm{~mL}$ blood loss within $24 \mathrm{~h}$ after vaginal delivery, or greater than $1,000 \mathrm{~mL}$ blood loss within $24 \mathrm{~h}$ after caesarean section), severe postpartum hemorrhage induces hemodynamic instability in pregnant women threatening their lives $(4,5)$. Treatment may include pelvic vascular embolism or hysterectomy. Hence, severe postpartum hemorrhage has attracted the attention of obstetricians. The definition of severe postpartum hemorrhage as stated in the Society of Obstetrics and Gynecology differs for each country (1-3). The Chinese Society of Obstetrics and Gynecology 
defines severe postpartum hemorrhage as postpartum bleeding volume reaching or over $1,500 \mathrm{~mL}$ and sets as grade predicting line to demarcate the highest danger level. It states that when postpartum blood loss is over this threshold, obstetricians should actively initiate multi-disciplinary treatment, timely transfer treatment and intensive care.

Placenta previa is the main reason for postpartum hemorrhage, although placenta previa does not always lead to severe postpartum hemorrhage (6). However, when combined with abnormal placenta implantation in the uterine wall, placenta previa may lead to severe postpartum hemorrhage, which requires emergent hysterectomy. This results in injury to the bladder or ureter and a significant increased risk of maternal and fetal death $(7,8)$. Hence, accurate evaluation of the risks of severe postpartum hemorrhage in placenta previa is critically important to reduce the mortality in pregnant women.

Imaging methods, such as ultrasound and MRI, are often used to evaluate the risk of severe postpartum hemorrhage in placenta previa (9). The sensitivity of placenta implantation evaluated using ultrasound is between 33$100 \%$, while its specificity is around $37.5-96 \%$ (10). MRI is often used in tertiary hospitals for further evaluation (11). Physicians can use these imaging methods to predict the risk of severe postpartum hemorrhage and prepare during the perioperative period to deal with possible complications.

However, not all physicians in China have the expertise to evaluate severe postpartum hemorrhage from placenta previa-related images, and not all hospitals have the resources to perform MRI. Evaluating imaging data requires a high degree of expertise. In addition, overlooking quantitative data can influence the accuracy of the evaluation. Hence it is clinically significant to establish a model to predict the risk of severe postpartum hemorrhage in patients with placenta previa. This will require patients' clinical and imaging data. In this study, risk factors that were correlated with severe postpartum hemorrhage in placenta previa were analyzed, then a model to predict severe postpartum hemorrhage derived from clinical and imaging information was established. This model could guide obstetricians to accurately evaluate severe bleeding and prepare treatment strategies for patients with severe postpartum hemorrhage.

\section{Methods}

\section{Study subjects}

Study subjects were women with placenta previa that gave birth from Jan. 2014 to Dec. 2017 in The First Affiliated Hospital of Army Medical University. During this period, there were a total of 22,732 premature or mature puerperas (28-42 weeks), among which there were 819 patients with placenta previa. Excluding patients with surgical diseases, fetal hypoplasia and incomplete clinical and ultrasound information, 435 patients met the inclusion criteria for this study.

\section{Diagnosis of placenta previa and severe postpartum bemorrhage}

Diagnosis of placenta previa resulting in pregnancy termination before 28 weeks is defined as miscarriage in China. Complete electronic patient information was extracted which included clinical and placental ultrasound images after 28 weeks of pregnancy.

Based on the Guideline for the Management and Prevention of Postpartum Hemorrhage issued by the Obstetrics and Gynecology of Chinese Medical Association, blood loss was evaluated using a combination of gauze weigh method and blood disc collection. In this study, postpartum blood loss was measured at the beginning of skin incision for caesarean section or at the beginning of perioperative vaginal bleeding for emergent caesarean section, or at the beginning of parturition for vaginal delivery. Measurements were obtained for $24 \mathrm{~h}$ after surgery or vaginal delivery. Blood loss over $1,500 \mathrm{~mL}$ was defined as severe postpartum hemorrhage, and was the only clinical observation in this study.

Diagnosis for placenta previa was performed after 28 weeks of pregnancy using vaginal ultrasonography. Placenta previa was defined as when the placenta reached or covered the intracervical mouth. Ultrasound diagnosis was verified by experienced ultrasound obstetricians $1-2$ weeks prior to pregnancy termination.

\section{Collection of clinical information}

Based on published guidelines and reviews (12-17), we collected clinical information related to postpartum hemorrhage, including age, residence, education level, assisted reproduction, history of pregnancy (number of pregnancies, times of delivery, times of miscarriage, history of vaginal delivery, history of caesarean section, history of uterine surgery, history of surgical complications, history of premature births, history of placenta previa), current pregnancy information (body mass index before pregnancy, 
number of pregnancy test, presence of uterine fibroids, anemia, thrombocytopenia, hypertension, or ICP; amniotic fluid index at last ultrasound; vaginal bleeding history during gestation period, volume of bleeding, treatment with magnesium sulfate, fetal cephalic presentation, gestational weeks at pregnancy termination, surgical anesthesia, vaginal blood loss, blood transfusion, etc.). Intravertebral anesthesia was used at our hospital, and switched to general anesthesia when needed.

\section{Collection of placenta information}

Based on guidelines and reviews (12-17), we collected placenta information related with postpartum hemorrhage, including placenta type (low-lying placenta, lowest site of placenta within $2 \mathrm{~cm}$ from the intracervical mouth; marginal placenta: placenta reaching the intracervical mouth; partial placenta: part of the placenta covering the intracervical mouth; complete placenta: placenta completely covering the intracervical mouth); placenta site (main body of placenta covering the anterior wall of the uterus, defined as the anterior wall of placenta, and that covering the posterior or lateral wall of the uterus defined as the posterior wall of the placenta); uterine placenta junction echo (spongy placenta; placental cleft appearance: 4 or more low-echo regions with a diameter greater than $5 \mathrm{~mm}$ in the placenta); placenta covering the uterine-bladder junction (uterine serosal line is clear and continuous, uterine serosal line is discontinuous or completely not visible); abundance of blood vessels at the uterine placenta junction viewed under Color Doppler. Depth of placenta invading the uterine muscle wall: based on vascular richness at echo of the uterine placenta junction, uterine bladder junction covered by the placenta, and uterine placenta junction. Correlation was preliminarily evaluated based on no abnormal implantation, placenta adhesion, placenta implantation and placenta penetration. Placenta previa was confirmed using surgical records that described the relationship between the placenta and mesometrium and post-surgical pathology.

\section{Statistical analysis}

Statistical analysis was performed using Windows SPSS 22.0 software. For descriptive statistics, quantitative data was represented as mean $\pm \mathrm{SD}$ (or $\mathrm{SE}$ ), and discrete data was represented as frequencies. Distribution index was evaluated using Kolmogorov-Smirnov with a cut-off of $\alpha=0.05$. Comparison of the quantitative index between groups was performed using an independent sample $t$ test or Mann-Whitney $U$ test, while qualitative index was analyzed using $2 \times 2$ contingency table or Kendall correlation analysis (non-parametric test).

Risk factors correlated with severe postpartum hemorrhage were obtained after all the variables were analyzed using univariate analysis. The $\mathrm{R}$ language programme was used to randomly sample at $8: 2(\mathrm{n}=377$ for the control group and $\mathrm{n}=98$ for the experimental group). They were then divided into the training set $(n=269$ for the control group, $\mathrm{n}=78$ for the experimental group) and the verification set $(n=68$ for the control group, $n=20$ for the experimental group). Independent risk factors were obtained using stepwise logistic regression analysis to establish the severe postpartum hemorrhage prediction model. In addition, discriminant boundary values of the model (probability) was performed. Receiver operating characteristic curve (ROC) was used to evaluate the effectiveness of the model. Area under curve (AUC) was used to determine the accuracy of the model.

\section{Results}

\section{General data}

A total of 435 patients were enrolled in our study, and included 29 patients who underwent vaginal delivery and 406 patients who underwent caesarean sections. The patients in the vaginal delivery group were those with lowlying placentas. Four of these patients had blood loss lower than $500 \mathrm{~mL}$, while 22 had blood loss that ranged between $500-1,499 \mathrm{~mL}$. Of the 7 patients who had blood loss of over $1,000 \mathrm{~mL}$ needed blood transfusion, while 3 patients with blood loss greater than $1,500 \mathrm{~mL}$ (severe postpartum hemorrhage) also had blood transfusions. None of the patients underwent hysterectomy. For the 406 patients who underwent caesarean section, 74 were diagnosed with lowlying placenta, 29 with marginal placenta previa, 9 with partial placenta previa, and 294 with central placenta previa. There were 222 patients with blood loss less than $1,000 \mathrm{~mL}$, 89 with blood loss ranging from $1,000-1,500 \mathrm{~mL}$ and 95 patients who had blood loss greater than $1,500 \mathrm{~mL}$ (severe postpartum hemorrhage). There were 194 patients who had blood transfusions while 10 patients had hysterectomies. No deaths were recorded for both mothers and newborns. Table 1 shows all the clinical parameters of each patients. 
Table 1 Clinical indices of patient population

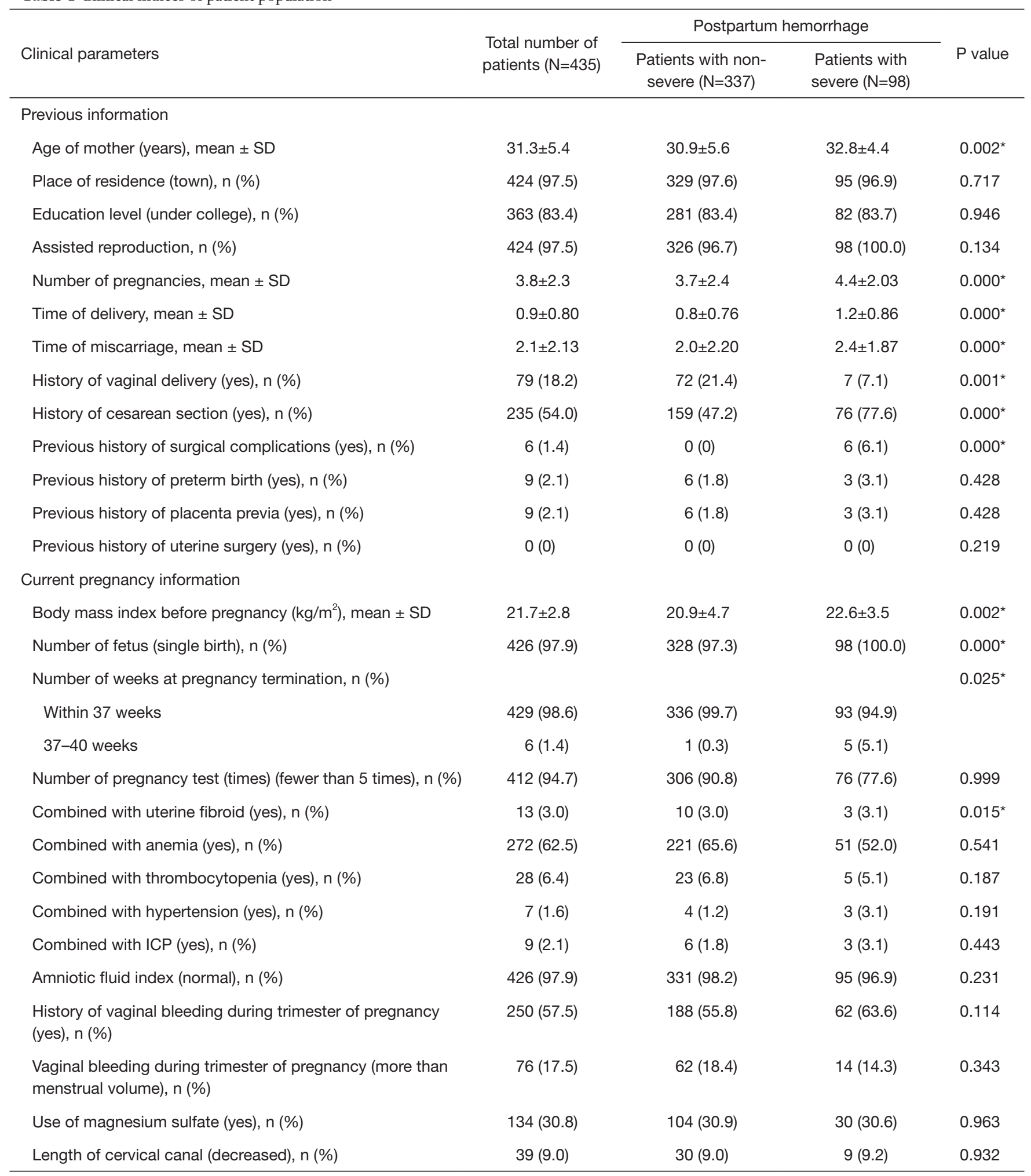

Table 1 (continued) 
Table 1 (continued)

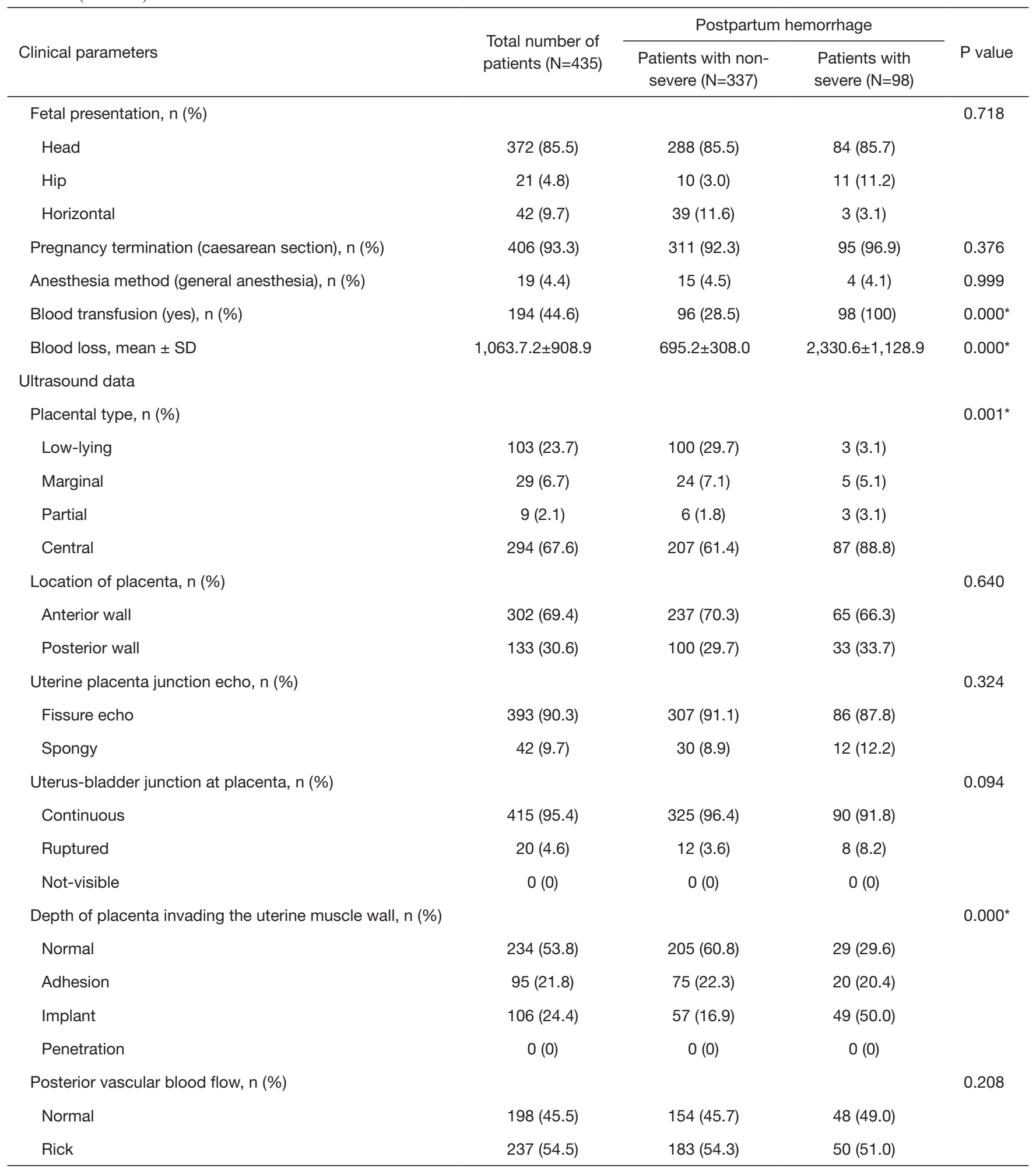

${ }^{*} \mathrm{P}<0.05$. 
Table 2 Regression coefficient estimation and test value $\hat{O R}$ of the risk model predicting severe postpartum hemorrhage in placenta previa

\begin{tabular}{|c|c|c|c|c|c|c|c|}
\hline \multirow{2}{*}{ Clinic variable } & \multirow{2}{*}{ b } & \multirow{2}{*}{ SE (b) } & \multirow{2}{*}{ Wald $\chi^{2}$} & \multirow{2}{*}{$\mathrm{P}$} & \multirow{2}{*}{$\hat{O R}$} & \multicolumn{2}{|c|}{$\hat{O R} 95 \% \mathrm{Cl}$} \\
\hline & & & & & & Lower & Upper \\
\hline Age (X1) & 0.075 & 0.030 & 2.465 & $0.013^{\star}$ & 1.016 & 1.091 & 1.291 \\
\hline Time of delivery (X2) & 1.531 & 0.217 & 7.061 & $0.000^{\star * *}$ & 3.087 & 1.889 & 5.197 \\
\hline Vaginal delivery (X4) & -3.557 & 0.593 & -5.993 & $0.000^{\star \star *}$ & 0.008 & 0.003 & 0.082 \\
\hline Gestational weeks at termination (X5) & 1.753 & 0.282 & 6.220 & $0.000^{\star \star \star}$ & 3.373 & 5.584 & 28.148 \\
\hline $\begin{array}{l}\text { Depth of placenta invading the uterine } \\
\text { muscle wall }(X 6)\end{array}$ & 1.574 & 0.165 & 9.552 & $0.000^{\star \star \star}$ & 3.537 & 2.961 & 7.072 \\
\hline
\end{tabular}

${ }^{*} \mathrm{P}<0.05,{ }^{\star \star *} \mathrm{P}<0.001$.

\section{Risk factor of severe postpartum bemorrbage}

Univariate analysis was used to analyze clinical and ultrasound imaging data. The results showed that age, time of pregnancy, time of delivery, time of miscarriage, history of vaginal delivery, history of caesarean section, surgical complications, body mass index before pregnancy, number of fetus, weeks at pregnancy termination, combined with uterine fibroid, type of placenta previa, depth of placenta invading into uterine muscle wall, and blood transfusions were significantly correlated with severe postpartum hemorrhage $(\mathrm{P}<0.05)$, as shown in Table 1.

\section{$A$ risk model to predict severe postpartum hemorrhage}

Stepwise logistic regression analysis of the 13 risk factors indicated that age, times of delivery, time of miscarriage, history of vaginal delivery, weeks at pregnancy termination, depth of placenta invading the uterine muscle wall, and blood transfusion during delivery were independent risk factors for severe postpartum hemorrhage (Table 2). We found that blood transfusions during delivery was an intraoperative variable and should be determined prior to delivery when including variables for model establishment. Therefore, it should be omitted at the time of establishing the model. Logistic regression prediction model was used to predict severe postpartum hemorrhage after random sampling using $\mathrm{R}$ language, with the discriminant boundary value of the model being established (probability: $P, 0.268$ ) $(0 \leq \mathrm{P} \leq 1)$. That is, after each variable is introduced into the model equation, if the probability calculated is higher than 0.268 , it denotes a higher occurrence of severe postpartum hemorrhage, while a lower than 0.268 denotes a lower probability.

Logistic risk prediction model equation was as follows: $\mathrm{P}=\log (\mathrm{Y} / 1-\mathrm{Y})$, where $\mathrm{Y}=-6.942+0.075 X_{1}($ age $)+1.531$ $X_{2}$ (times of delivery) $+0.223 X_{3}$ (time of miscarriage) $3.557 X_{4}$ (vaginal delivery: 1 for yes, 0 for no) $+1.753 X_{5}$ ( 0 for $<37$ weeks, 1 for $\geq 37$ weeks) $+1.574 X_{6}$ (relationship between placenta and uterine muscle wall: 0 for normal, 1 for placenta adhesion, 2 for placenta implantation, 3 for placenta penetration).

For example, a pregnant woman aged 29 years $\left(X_{1}=29\right)$, delivered once $\left(X_{2}=1\right)$, miscarriage once $\left(X_{3}=1\right)$, vaginal delivery $0\left(X_{4}=0\right)$, gestational weeks at pregnancy termination $36+5$ weeks $\left(X_{5}=0\right)$, normal in placenta $\left(X_{6}=0\right)$, $\mathrm{Y}=-6.942+0.075 \times 29+1.531 \times 1+0.223 \times 1-3.557 \times 0+$ $1.753 \times 0+1.574 \times 0=-3.016$, Y was inserted into logical equation to obtain $\mathrm{P}=0.0467<0.268$, indicating a lower possibility to have severe postpartum hemorrhage.

\section{Effectiveness of the logistic risk prediction model}

To verify the effectiveness of the logistic risk prediction model equation, 88 patients were randomly selected from the total patient population $(n=435)(0$, no severe postpartum hemorrhage $n=68 ; 1$, severe postpartum hemorrhage, $n=20$ ) to determine the verification rate. The results showed that sensitivity was $\left(\widehat{S}_{e}\right)=0.765$ (negative prediction accuracy rate), specificity $\left(\hat{S}_{p}\right)=0.900$ (positive prediction accuracy rate), and total accuracy rate was 0.795 . Based on the validation results, AUC of ROC was 0.8419 , as shown in Figure 1 and Table 3. 


\section{Discussion}

Placenta previa is a major reason for severe postpartum hemorrhage (12-18). With the increase in caesarean sections worldwide, as well as the incidence of placenta previa, a significant increase in severe postpartum hemorrhage has been documented $(19,20)$. Predicting accurately the occurrence of severe postpartum hemorrhage in placenta previa patients in advance will be helpful to reduce maternal and fetal mortality. The established model that we generated predicted severe postpartum hemorrhage in placenta previa patients based on independent risk factors of severe postpartum hemorrhage, and included clinical and ultrasound imaging data. The sensitivity and specificity of the model was $76.5 \%$ and $90.0 \%$, respectively. Using this model physicians could effectively use it to determine the risks of severe postpartum hemorrhage in placenta previa patients.

The definition on severe postpartum hemorrhage as stated in the Society of Obstetrics and Gynecology differs between countries (1-3). The definition of blood loss in severe postpartum hemorrhage varies between the different countries' guidelines, i.e., over 1,500 mL (17), 2,000 mL (17), $30-40 \%$ of whole blood volume (20), or over 4 units of blood transfusion $(800 \mathrm{~mL})(6)$. In this study, severe

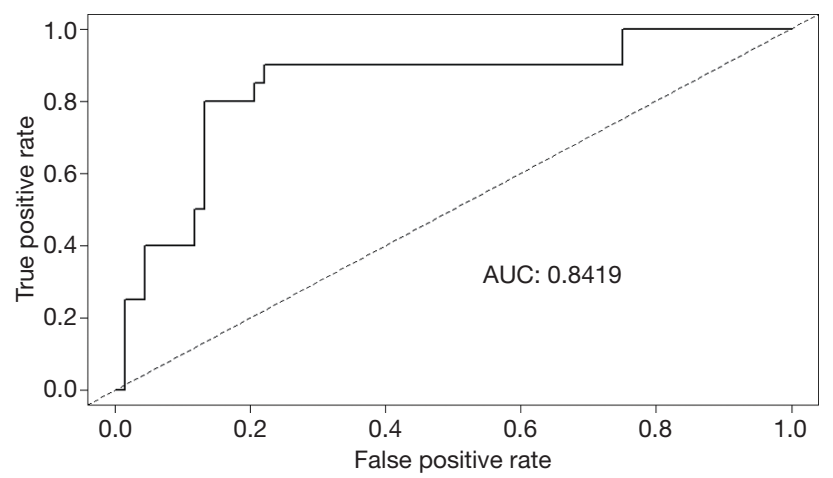

Figure 1 ROC curve of the risk prediction model. postpartum hemorrhage was defined as blood loss over 1,500 mL. The Management and Prevention of Postpartum Hemorrbage published by the Gynecology and Obstetrics of Chinese Medical Association (19), states that when postpartum blood loss over 1,500 mL occurs, special measures should be implemented to save the patients' life. This includes timely referrals, MDT joint treatment, uterine artery embolism, or panhysterectomy, as well as intensive care in ICU. We did not define postpartum hemorrhage based on blood transfusion volume, because it is associated with basal hemoglobin of puerpera (17).

In this study, risk factors for severe postpartum hemorrhage included age, number of pregnancies, number of deliveries, number of miscarriages, history of vaginal delivery, history of caesarean section, surgical complications, body mass index before pregnancy, number of fetus, weeks at pregnancy termination, presence of uterine fibroids, placenta previa types, depth of placenta invading into the uterine muscle wall, and blood transfusions. Stepwise logistic regression analysis indicated that age, number of deliveries, number of miscarriages, history of vaginal delivery, weeks at pregnancy termination, correlation between placenta and uterine muscle wall, and blood transfusion during delivery were independent risk factors for severe postpartum hemorrhage. However, these risk factors differed from other previous studies $(16,21)$.

In this study, we found that age was an independent risk factor for severe postpartum hemorrhage of placenta previa $(\mathrm{OR}=1.078)$ (18). Elderly pregnant women have reduced reproductive function (greater than 40 years old was defined as elderly pregnant women in our study) and may be accompanied with miscarriages or uterine surgical history. The possibility of complicated pregnancies is high, and the risk of severe postpartum hemorrhage is also increased (22). In addition, multiple deliveries are considered risk factors for postpartum hemorrhage (23). Time of deliver has also been proven to be an independent risk factor for severe postpartum hemorrhage with OR of 4.635. Since multiple

Table 3 Results of validating the risk prediction model

\begin{tabular}{|c|c|c|c|c|c|c|}
\hline $\begin{array}{l}\text { Actual } \\
\text { category }\end{array}$ & \multicolumn{2}{|c|}{ Prediction category } & Total & $\begin{array}{l}\text { Negative prediction } \\
\text { accuracy rate (\%) }\end{array}$ & $\begin{array}{l}\text { Positive prediction } \\
\text { accuracy rate (\%) }\end{array}$ & $\begin{array}{l}\text { General prediction } \\
\text { accuracy rate (\%) }\end{array}$ \\
\hline 0 & 52 & 16 & 68 & $76.5(52 / 68)$ & - & - \\
\hline 1 & 2 & 18 & 20 & - & $90.0(18 / 20)$ & - \\
\hline Total & 54 & 34 & 88 & - & - & 79.5 (70/88) \\
\hline
\end{tabular}


deliveries could lead to changes in contraction in the mesometrium, it is hypothesized to be a significant factor for severe postpartum hemorrhage (23).

High incidence of induced abortions and miscarriages are worldwide problems (24). Repeated miscarriages significantly increased the risk of postpartum hemorrhage $(17,19)$. Our study demonstrated that the time of miscarriage was an independent risk factor for severe postpartum hemorrhage in placenta previa $(\mathrm{OR}=1.250)$. Repeated miscarriage leads to injury of the endometrium, and the probability of abnormal placenta previa implantation is significantly increased, leading to severe postpartum hemorrhage $(11,25)$.

We found correlations with caesarean sections and uterine surgical complications with severe postpartum hemorrhage in placenta previa, which was consistent with a previous study (26). Histories of caesarean sections and uterine surgical complications are considered risk factors for the occurrence of placenta previa implantation, and is frequently associated with severe postpartum hemorrhage (26). In addition, body mass index before pregnancy, number of fetuses, and the presence of uterine fibroids are associated with severe postpartum hemorrhage (27), but were not independent risk factors for severe postpartum hemorrhage. Interestingly, we found that vaginal delivery history was an independent protective factor for severe postpartum hemorrhage $(\mathrm{OR}=0.029)$. This could be explained by the cervix uteri being relaxed after vaginal delivery. However, the contraction ability of the lower uterine segment becomes weakened with additional deliveries and the risk of postpartum hemorrhage increases (25). There are no published reports to explain this observation.

At the time of emergent caesarean section, risk of severe postpartum hemorrhage is significantly higher compared to selective cesarean section and is associated with emergent hysterectomy (28). Emergent caesarean section is often closely related with uterine tear, macrovascular injury and unskilled surgeons, which may result in severe postpartum hemorrhage (13). Complete perioperative preparation will be helpful to reduce the occurrence of severe postpartum hemorrhage and related complications (13). Hence, selective caesarean section before and after 37 weeks of pregnancy is often preferred for pregnancy termination in placenta previa. In this study, we found that gestational weeks of pregnancy termination was an independent risk factor for severe postpartum hemorrhage $(\mathrm{OR}=5.774)$. Pregnancy termination after 37 weeks increases the risk of severe postpartum hemorrhage and may be related with the higher possibility of urgent caesarean section (29).

Complete placenta previa is a risk factor for severe postpartum hemorrhage (30,31). More placenta covering the intracervical mouth the higher risk of bleeding during the perioperative period (6). Partial placenta previa covering the intracervical mouth is also a risk factor for severe postpartum hemorrhage (30). Placental vessels penetrate other blood vessels supplying the bladder wall adjacent to the lower anterior region of the uterus which will result in massive bleeding during the separation of the placenta from the uterus when the placenta is located anteriorly (6). Based on this, the placenta previa at the anterior wall is more likely to result in severe postpartum hemorrhage. However, comparisons between different implantation of the placenta previa suggests that placenta previa located in the posterior wall of the uterus is no different to that located in the anterior for the occurrence of severe postpartum hemorrhage (32).

An abnormal invasion of chorionic villi into the myometrium leads to Placenta Accreta spectrum. This includes the attachment of the placenta to the myometrium without intervening decidua (placenta accreta), the invasion of the myometrium (placenta increta), and the infiltration of the surrounding organs into the uterine serosa (placenta percreta) (33). The average blood loss at delivery in women with Placenta Accreta spectrum is reported to be over $3,000 \mathrm{~mL}$ and will require blood transfusion in $90 \%$ of patients (21). Our results indicate that the association between the placenta and the uterine muscle wall is the highest independent risk factor for severe postpartum hemorrhage $(\mathrm{OR}=4.825)$. The incidence of placenta implantation increases with the number of caesarean sections (7). Placenta implantation is considered the main reason for intrapartum hysterectomy (34) and has attracted increased attention from Society of Obstetrics and Gynecology worldwide. For placenta previa (especially suspicious abnormal placenta implantation), the RANZOG and SOGC guidelines recommend vigilant prenatal evaluation and transfer to tertiary medical centers with blood transfusion and intensive care facilities. ACOG and RCOG guidelines recommend pregnant women should consider the possibility of blood transfusions, hysterectomy or termination of their pregnancies.

None of the guidelines specify a preferred modality for evaluating abnormal placentation. However, there are multiple investigations trying to predict severe postpartum hemorrhage risk by analyzing imaging data of placenta previa. Ultrasound is the primary tool to diagnose AIP in 
women at risk, such as those with placenta previa and with prior cesarean sections (35). Ultrasound had an overall good diagnostic accuracy in identifying the depth of placental invasion with sensitivities of $90.6 \%, 93.0 \%, 89.5 \%$, and $81.2 \%$ for placenta accreta, increta, accreta/increta, and percreta respectively (10). Several ultrasound features have been suggested for the diagnosis of placenta accrete, such as irregularly shaped placental lacunae (vascular spaces), thinning of the myometrium overlying the placenta, loss of the retroplacental "clear space", protrusion of the placenta into the bladder, increased vascularity of the uterine serosa/ bladder interface, and turbulent blood flow through the lacunae on Doppler ultrasonography (36). However, using a single ultrasound image to predict the risk of severe postpartum hemorrhage lacks an objective evaluation standard. Diagnostic accuracy depends on the training and experience level of the physician, irrespective of the imaging technique (35). A growing interest towards the use of magnetic resonance (MR) imaging for evaluating placenta previa has been observed (10). MRI could be helpful in adding topographical and morphological information and represents a complementary tool for patients with equivocal ultrasound findings or when additional information is required (37). However, analyzing MRI images requires professional training, which limits its promotion and application for predicting severe postpartum hemorrhage in placenta previa.

For predicting the risk of severe postpartum hemorrhage in placenta previa, experienced obstetricians could make a judgement using the relevant clinical factors and imaging data (38), and get prepared in advance for the occurrence of severe complications, such as severe postpartum hemorrhage, and emergent hysterectomy (21). However, not all the obstetricians in China are clinically experienced to determine these risks. Hence, it is critical to establish a model to accurately predict severe postpartum hemorrhage in placenta previa patients. After analyzing relevant risk factors, our prediction model was established to include clinical and imaging data. The establishment of the prediction model for severe postpartum hemorrhage in placenta previa is based on subjective assessments of risk factors from clinical experience, and through a scoring system established after risk factor OR determination $(15,16)$. Conversely, independent risk factors were analyzed in R to ensure objectivity and accuracy of the model. Risk factor variables contained in the model were objective and hence reduced bias. Even if abnormal placenta implantation was not determined preoperatively, it could be corrected intraoperatively.

Limitations of the study were as follows; first, this was a single center study and may not be representative and the clinical strategies may not completely correspond with established guidelines. Second, this study was a retrospective in nature. We observed that clinical information for some patients were missing or lacked detail, i.e. observation of adherent placenta and placenta implantation but without details of placental penetration. In addition, some of the ultrasounds were of poor quality and were omitted from our study. However, the prediction model we established for severe postpartum hemorrhage in placenta previa had high sensitivity and specificity and is appropriate for clinical application.

Taken together, by analyzing data from placenta previa in the last 4 years in our center, independent risk factors for severe postpartum hemorrhage in placenta previa were established and were used in our model. Based on clinical information, the model reduced subjective dependence on clinical experience and had predictive accuracy, and hence could be clinically applicably. However, our predictive model needs to be validated in other clinical institutions. Meanwhile, we will prospectively collect additional clinical patient data to supplement our prediction model to enhance its accuracy. We believe our model will help surgeons prepare and reduce severe bleeding and mortality in pregnant women with placenta previa.

\section{Acknowledgments}

Funding: This study was supported by Social People's Livelihood Scientific and Technological Innovation Special Project (No. cstc2015shmszx120065).

\section{Footnote}

Conflicts of Interest: The authors have no conflicts of interest to declare.

Ethical Statement: The authors are accountable for all aspects of the work in ensuring that questions related to the accuracy or integrity of any part of the work are appropriately investigated and resolved. The study was supported by grants from the Chongqing Science and Technology Commission (CSTC2015SHMSZX120065) and approved by the Ethics Committee of The First Affiliated Hospital of the Army Medical University (2007-11). 


\section{References}

1. ACOG Practice Bulletin: Clinical Management Guidelines for Obstetrician-Gynecologists Number 76, October 2006: postpartum hemorrhage. Obstet Gynecol 2006;108:1039-47.

2. Clinical Practice Obstetrics Committee. Active management of the third stage of labour: prevention and treatment of postpartum hemorrhage: No. 235 October 2009 (Replaces No. 88, April 2000). Int J Gynaecol Obstet 2010;108:258-67.

3. Tunçalp O, Souza JP, Gulmezoglu M. New WHO recommendations on prevention and treatment of postpartum hemorrhage. Int J Gynaecol Obstet 2013;123:254-6.

4. Creanga AA, Berg CJ, Syverson C, et al. Pregnancyrelated mortality in the United States, 2006-2010. Obstet Gynecol 2015;125:5-12.

5. Grobman WA, Bailit JL, Rice MM, et al. Frequency of and factors associated with severe maternal morbidity. Obstet Gynecol 2014;123:804-10.

6. Lee HJ, Lee YJ, Ahn EH, et al. Risk factors for massive postpartum bleeding in pregnancies in which incomplete placenta previa are located on the posterior uterine wall. Obstet Gynecol Sci 2017;60:520-6.

7. Silver RM. Abnormal Placentation: Placenta Previa, Vasa Previa, and Placenta Accreta. Obstet Gynecol 2015;126:654-68.

8. Timor-Tritsch IE, Monteagudo A. Unforeseen consequences of the increasing rate of cesarean deliveries: early placenta accreta and cesarean scar pregnancy. A review. Am J Obstet Gynecol 2012;207:14-29.

9. Riteau AS, Tassin M, Chambon G, et al. Accuracy of ultrasonography and magnetic resonance imaging in the diagnosis of placenta accreta. PLoS One 2014;9:e94866.

10. Pagani G, Cali G, Acharya G, et al. Diagnostic accuracy of ultrasound in detecting the severity of abnormally invasive placentation: a systematic review and meta-analysis. Acta Obstet Gynecol Scand 2018;97:25-37.

11. Einerson BD, Rodriguez CE, Kennedy AM, et al. Magnetic resonance imaging is often misleading when used as an adjunct to ultrasound in the management of placenta accreta spectrum disorders. Am J Obstet Gynecol 2018;218:618.e1-7.

12. Butwick AJ, Ramachandran B, Hegde P, et al. Risk Factors for Severe Postpartum Hemorrhage After Cesarean Delivery: Case-Control Studies. Anesth Analg 2017;125:523-32.
13. Ekin A, Gezer C, Solmaz U, et al. Predictors of severity in primary postpartum hemorrhage. Arch Gynecol Obstet 2015;292:1247-54.

14. Green L, Knight M, Seeney FM, et al. The epidemiology and outcomes of women with postpartum haemorrhage requiring massive transfusion with eight or more units of red cells: a national cross-sectional study. Bjog 2016;123:2164-70.

15. Kim JW, Lee YK, Chin JH, et al. Development of a scoring system to predict massive postpartum transfusion in placenta previa totalis. J Anesth 2017;31:593-600.

16. Lee JY, Ahn EH, Kang S, et al. Scoring model to predict massive post-partum bleeding in pregnancies with placenta previa: A retrospective cohort study. J Obstet Gynaecol Res 2018;44:54-60.

17. Nyfløt LT, Sandven I, Stray-Pedersen B, et al. Risk factors for severe postpartum hemorrhage: a case-control study. BMC Pregnancy Childbirth 2017;17:17.

18. Lal AK, Hibbard JU. Placenta previa: an outcome-based cohort study in a contemporary obstetric population. Arch Gynecol Obstet 2015;292:299-305.

19. Obstetrics Subgroup, Chinese Society of Obstetrics and Gynecology, Chinese Medical Association; Obstetrics Subgroup Chinese Society of Obstetrics and Gynecology Chinese Medical Association. Guideline of prevention and treatment about postpartum hemorrhage (2014). Zhonghua Fu Chan Ke Za Zhi 2014;49:641-6.

20. Sebghati $M$, Chandraharan E. An update on the risk factors for and management of obstetric haemorrhage. Womens Health (Lond) 2017;13:34-40.

21. Duzyj CM, Cooper A, Mhatre M, et al. Placenta Accreta: A Spectrum of Predictable Risk, Diagnosis, and Morbidity. Am J Perinatol 2019;36:1031-8.

22. Durmaz A, Komurcu N. Relationship Between Maternal Characteristics and Postpartum Hemorrhage: A MetaAnalysis Study. J Nurs Res 2018;26:362-72.

23. Dahlke JD, Mendez-Figueroa H, Maggio L, et al. Prevention and management of postpartum hemorrhage: a comparison of 4 national guidelines. Am J Obstet Gynecol 2015;213:76.e1-10.

24. Ngo TD, Keogh S, Nguyen TH, et al. Risk factors for repeat abortion and implications for addressing unintended pregnancy in Vietnam. Int J Gynaecol Obstet 2014;125:241-6.

25. Eshkoli T, Weintraub AY, Sergienko R, et al. Placenta accreta: risk factors, perinatal outcomes, and consequences for subsequent births. Am J Obstet Gynecol 2013;208:219. e1-7. 
26. Kramer MS, Berg C, Abenhaim H, et al. Incidence, risk factors, and temporal trends in severe postpartum hemorrhage. Am J Obstet Gynecol 2013;209:449.e1-7.

27. Briley A, Seed PT, Tydeman G, et al. Reporting errors, incidence and risk factors for postpartum haemorrhage and progression to severe $\mathrm{PPH}$ : a prospective observational study. Bjog 2014;121:876-88.

28. Giambattista E, Ossola MW, Duiella SF, et al. Predicting factors for emergency peripartum hysterectomy in women with placenta previa. Arch Gynecol Obstet 2012;285:901-6.

29. Bao Y, Xu C, Qu X, et al. Risk factors for transfusion in cesarean section deliveries at a tertiary hospital. Transfusion 2016;56:2062-8.

30. Pivano A, Alessandrini M, Desbriere R, et al. A score to predict the risk of emergency caesarean delivery in women with antepartum bleeding and placenta praevia. Eur J Obstet Gynecol Reprod Biol 2015;195:173-6.

31. Sekiguchi A, Nakai A, Kawabata I, et al. Type and location of placenta previa affect preterm delivery risk related to antepartum hemorrhage. Int J Med Sci 2013;10:1683-8.

32. Belachew J, Eurenius K, Mulic-Lutvica A, et al. Placental location, postpartum hemorrhage and retained placenta in women with a previous cesarean section delivery: a

Cite this article as: Chen C, Liu X, Chen D, Huang S, Yan X, Liu H, Chang Q, Liang Z. A risk model to predict severe postpartum hemorrhage in patients with placenta previa: a single-center retrospective study. Ann Palliat Med 2019;8(5):611621. doi: 10.21037/apm.2019.09.04 prospective cohort study. Ups J Med Sci 2017;122:185-9.

33. Silver RM, Branch DW. Placenta Accreta Spectrum. N Engl J Med 2018;378:1529-36.

34. D'Arpe S, Franceschetti S, Corosu R, et al. Emergency peripartum hysterectomy in a tertiary teaching hospital: a 14-year review. Arch Gynecol Obstet 2015;291:841-7.

35. Collins SL, Ashcroft A, Braun T, et al. Proposal for standardized ultrasound descriptors of abnormally invasive placenta (AIP). Ultrasound Obstet Gynecol 2016;47:271-5.

36. Calì G, Giambanco L, Puccio G, et al. Morbidly adherent placenta: evaluation of ultrasound diagnostic criteria and differentiation of placenta accreta from percreta. Ultrasound Obstet Gynecol 2013;41:406-12.

37. Delli Pizzi A, Tavoletta A, Narciso R, et al. Prenatal planning of placenta previa: diagnostic accuracy of a novel MRI-based prediction model for placenta accreta spectrum (PAS) and clinical outcome. Abdom Radiol (NY) 2019;44:1873-82.

38. Baba Y, Ohkuchi A, Usui R, et al. Calculating probability of requiring allogeneic blood transfusion using three preoperative risk factors on cesarean section for placenta previa. Arch Gynecol Obstet 2015;291:281-5. 